\title{
ERFASSUNG, BEHANDLUNG UND WIEDERVERWENDUNG VON GRAU- UND REGENWASSER IN EINFAMILIENHÄUSERN
}

\section{REVIEW ARTICLE}

MONTEIRO, Otávio Pinetti ${ }^{1}$

LIMA, Gemael Barbosa ${ }^{2}$

MONTEIRO, Otávio Pinetti. LIMA, Gemael Barbosa. Erfassung, Behandlung und Wiederverwendung von Grau- und Regenwasser in Einfamilienhäusern. Revista Científica Multidisciplinar Núcleo do Conhecimento. Jahrgang 05, Ed. 01, Band 01, S. 98-113. Januar 2020. ISSN:

2448-0959,

Zugangslink: https://www.nucleodoconhecimento.com.br/umgebung/aschewasser

\section{ZUSAMMENFASSUNG}

Ziel dieser Arbeit ist es, eine bibliographische Übersicht zu präsentieren, deren Thema die Wiederverwendung von Wasser durch Regen sowie die Wiederverwendung von grauer Wasser in einem Einfamilienhaus ist. Dazu stellt die Methodik eine Informationssuche in Büchern, Zeitschriften, Artikeln, Masterarbeiten und Dissertationen dar. Zur bewussten Wiederverwendung von Möglichkeiten, Wasser aus einem Wohnhaus zu fangen, sei es aus dem Regen, der auf die Dachrinnen fällt oder in den Methoden der Erfassung in den Küchenspülen und/oder Tanks von Servicebereichen verwendet wird, die die notwendige Behandlung erhalten und wiederverwendet werden. Diese Behandlungen wirken effizient und bieten die

1 Postgraduate in Environmental Engineering and Occupational Safety (Faveni), Postgraduate in Sustainability in the Built Environment (Ifes), Post-Graduate in Teaching of Higher Education (Faveni), Graduateed in Civil Engineering (Unesc), Graduating in Mathematics.

${ }^{2}$ Master in Environmental Engineering, Professor am UNESC. 
Verwendung dieses Wassers für weniger edle Zwecke, die den Verbrauch rationaler und wirtschaftlicher macht, zusammenarbeite für die Erhaltung der Umweltressourcen.

Stichworte: Wasser, Wiederverwendung, Gefangennahme, Behandlung, Wohnort.

\section{EINFÜHRUNG}

Wie Zampieron (2005) feststellt, ist Wasser zu einem gemeinsamen Symbol der Menschheit geworden, das in allen Religionen und Kulturen geschätzt wird, auch ein Symbol der sozialen Gleichheit, denn die Wasserkrise ist vor allem Verteilung, Ressourcen und nicht nur in ihrer absoluten Knappheit. . Mit dem klaren Ziel, den Wasserverbrauch durch neue Technologien zu senken, begann die Entwicklung mehrerer rationeller Nutzungsprogramme.

Diese damit verbundenen Probleme bestätigen die Diskussion über die Durchführbarkeit der Verwendung von Regenwasser und Grauwasser für grundlegende Aktivitäten in einem Wohnhaus. Diese Möglichkeit der Wiederverwendung wird sicherlich zu einem Rückgang des Verbrauchs von behandeltem Wasser führen, der von sanitären Einrichtungen bereitgestellt wird, außerdem wird die Nachfrage nach Kosten durch die Nutzung von Trinkwasser und die Verringerung der Risiken, die sich aus den Abwasserentsorgungsunternehmen ergeben, verringert. Überschwemmungen in Zeiten von Starkregen.

Die angesprochene Studie soll daher anhand einer Fallstudie zeigen, wie die Wiederverwendung von Wasser aus Regen zusammen mit der Wiederverwendung von Grauwasser in einem Einfamilienhaus erfolgt. Die gesamte Grundlage der Arbeit wurde durch Informationen in Büchern, Zeitschriften, Artikeln und Masterarbeiten gefunden, in denen die Autoren sowie Forscher aus der Region, Labor und wissenschaftliche Forschung verwendet wurden, um ihre Ergebnisse zu unterstützen.

Obwohl Wasser eine erneuerbare Ressource ist, entspricht sein Angebot nicht der Nachfrage, die im Laufe der Zeit wesentlich zugenommen hat. Damit wird es immer ein Defizit geben, das durch irrationale Nutzung und diverse Verschwendung immer 
mehr noch verstärkt wird. Daher wird es immer notwendiger, eine neue Beziehung zwischen Mensch und Wasser herzustellen, denn dies werden erweitert die Möglichkeiten der rationellen Nutzung und die Hinzufügung zu diesem Faktor, Alternativen, um effizient zu nutzen, ob durch Diefung und Behandlung von bei der Arbeit oder durch spezifischere Methoden, die von der Bevölkerung noch nicht gut aufgenommen werden, weil sie den sehr hohen Investitionspreis haben.

\section{THEORETISCHEN RAHMEN}

[...] In Kombination mit der vorgenannten Auflösungskapazität fungiert Wasser als Transportmittel - im Oberflächen- und Untertageablauf - und ermöglicht es, die Eigenschaften desselben Wasserverlaufs zeitlich und räumlich zu verändern. (LEBANON, 2010, S.15)

Die Potabilisierung natürlicher Gewässer für den menschlichen Verzehr hat als wesentliche Funktion die Anpassung von Rohwasser an die physikalischen, chemischen, biologischen und radioaktiven Grenzwerte gemäß der Verordnung 2914, wodurch die Abwässer der Station keine für die versorgte Bevölkerung schädlich.

[...] Die Wasseraufbereitung umfasst die Entfernung von schwebbaren und kolloidalen Partikeln, organischen Stoffen, Mikroorganismen und anderen schädlichen Stoffen zur menschlichen Gesundheit, die möglicherweise in natürlichen Gewässern vorhanden sind, zu den niedrigsten Kosten für Implantation, Betrieb und Wartung und die geringsten Umweltauswirkungen auf die umliegenden Gebiete zu erzeugen. (LIBÂNIO, 2010, p.135).

\subsection{NUTZUNG VON WASSER IM RAHMEN DES TIEFBAUS}

Laut Mayok (2009) gibt es andere Normen, wie NBR 7229/1993, das die Größe des Wassersystems hat; und die Richtlinien für die Erhaltung und Wiederverwendung von Wasser in Gebäuden, die von der Civil Construction Union (Sinduscon) erstellt wurden, 
die mehrere Möglichkeiten zur Wiederverwendung von Wasser bietet, auch beim Waschen von Zuschlagstoffen für den Einsatz im Tiefbau.

Laut Sickermann (2005), der von PROSAB zitiert wird, wird 2006 als äußerst wichtige Ressource für den Bau Wasser in allen Phasen eines Werkes genutzt, von der Herstellung von Beton bis hin zur Deckung der Grundbedürfnisse der Arbeiter - und seine Knappheit ist bereits in Baustellen an verschiedenen Standorten im Land.

Laut dem Vizepräsidenten der Baugewerkschaft (Sinduscon-MG) Geraldo Jardim Linhares Jénio, obwohl die Situation noch nicht ernst ist, haben sich Bauunternehmen seit einiger Zeit dafür entschieden, konventionelle Methoden durch Trockenbaumethoden zu ersetzen. , um eine gewisse Unabhängigkeit vom Wasser zu schaffen (MARIANE, 2014).

\subsection{MEHRFACHE VERWENDUNG VON WASSER}

Basierend auf der Forschung von Tomaz (2005) im Hinblick auf die Wiederverwendung von Regenwasser, ist der Einsatz von Maßnahmen und Techniken ausgezeichnet, um Probleme über den Mangel an Trinkwasser zu vermeiden und zu beheben, auch ohne Gesetze, die seine Verwendung regulieren. Der Einsatz geeigneter Methoden und die abwechselnde Verwendung mit Wasser aus haushaltskanälen wäre eine intelligente Möglichkeit, Wasser für edle Zwecke zu sparen.

Wie caubet (2006), Vertreter von Nichtregierungsorganisationen (NRO) im Nationalen Rat für Wasserressourcen (CNRH) angegeben, auch wenn die Wasserordnung (Bundesdekret Nr. 24.643, vom 10.07.1934) stellt die absolute Priorität der Verwendung von Wasser für die Grundbedürfnisse des Menschen (Verdeintung und Haushaltsnutzung) dar, der größte Rückzug findet im Bereich der Landwirtschaft statt (62,7\%), dann erfolgt durch den menschlichen Verzehr (17,9\%) und industrielle Nutzung (14\%) und schließlich beim Tierischen Verbrauch (5,4\%), wie in Abbildung 01 dargestellt. 
Abbildung 01-Wasserverbrauch pro Aktivität in Brasilien.

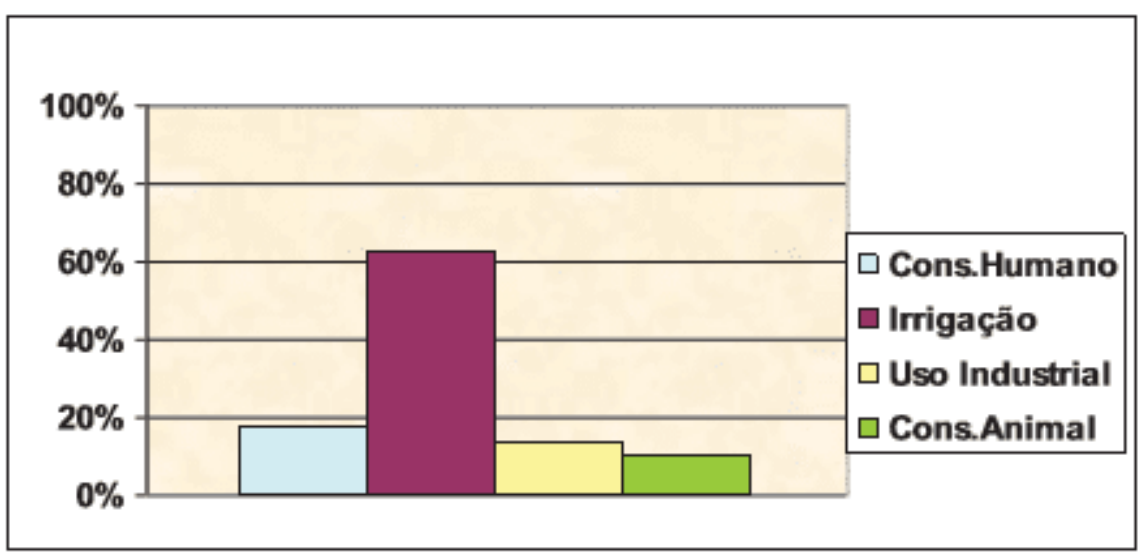

Quelle: ANA (2002) zitiert von MANUAL FIESP/CIESP (2006).

\subsubsection{VERWENDUNG VON NICHT-TRINKWASSER}

Studien in Brasilien und im Ausland zeigen, dass in einer Residenz, ein Großteil des Wasserverbrauchs konzentriert sich auf Bäder, die Entladung von Toiletten, Küchenspüle und Wäsche waschen. Im Durchschnitt werden $40 \%$ des gesamten In einem Wohnhaus verbrauchten Wassers nicht getrunken. (GONÇALVES, 2009).

Die Notwendigkeit, die Menge des Wassers zu analysieren, um grundlegende Aktivitäten des Verbrauchs und der Hygiene zu erfüllen, ist ein Grund für Diskussionen unter mehreren Autoren, wie wir durch Abbildung 02 analysieren können. Eine von Peter Gleick vorgeschlagene Definition, die in der Grundwasseranforderung (BWR) enthalten ist, besagt, dass $50 \mathrm{~L} / \mathrm{hab} / \mathrm{Tag}$ eine ausreichende Menge ist, um diesen Bedarf zu decken (BIO, 2002). 
Abbildung 02 - Verteilung des Wasserverbrauchs in den Haushalten nach Geräten

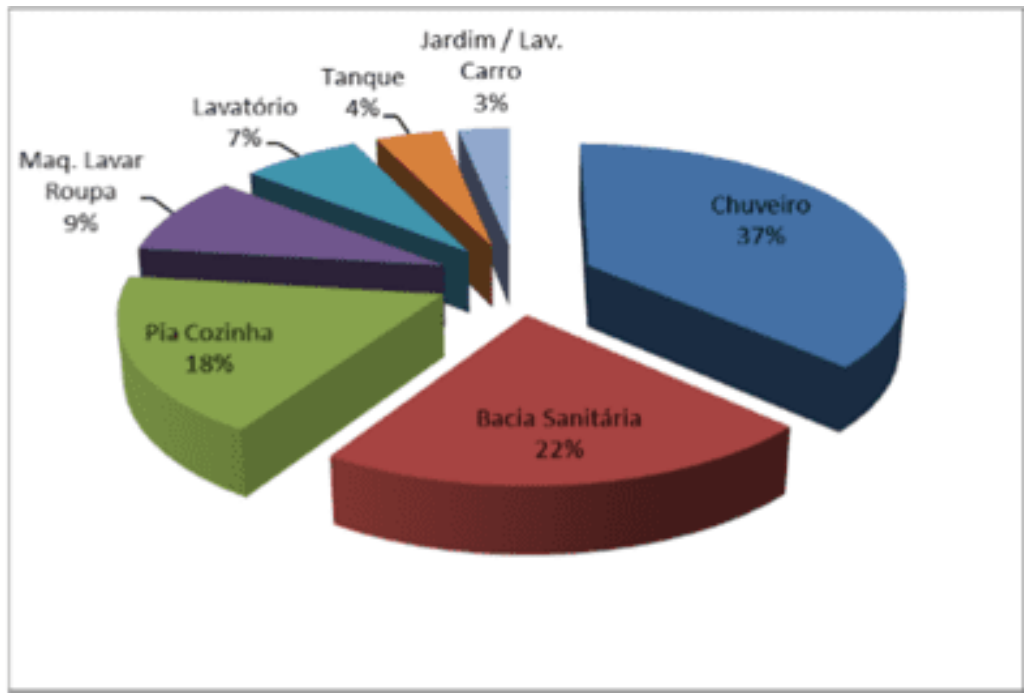

Quelle: Adapted from Martins and Memelli (2011)

\subsubsection{VERWENDUNG VON TRINKWASSER}

Trinkwasser ist alles Wasser, das für den Verbrauch geeignet ist. Eine farblose, geruchlose, geschmacks- und knochenlose Flüssigkeit zu sein, die für das menschliche Überleben unerlässlich ist, wie Hespanhol (2002) feststellt.

\subsubsection{BEHANDLUNG}

Es ist nicht unbedingt notwendig, dass das Wasser im Frühjahr alle geeigneten Indikatoren für den Verbrauch enthält, denn laut Braga (2005) gibt es Möglichkeiten, seine Eigenschaften zu ändern, um es mit den Anforderungen der öffentlichen Gesundheit in Einklang zu bringen.

Auch nach Braga (2005) sind die wichtigsten Methoden der Wasseraufbereitung: Dekantieren, Flocken, Filtration, Desinfektion, Entfernung von Härte, Belüftung, Entfernung von Eisen und Mangan, Entfernung von Geschmack und Geruch, Korrosionskontrolle und Fluoridierung, die immer und dass die fehlende angemessene Behandlung einiger dieser Gegenstände die endgültige Qualität des Wassers verändern kann. 


\section{MATERIALIEN UND METHODEN}

Die Methodik dieses Artikels bestand aus bibliographischer Forschung in Büchern, wissenschaftlichen Zeitschriften, Dissertationen und Masterarbeiten. Die Überprüfung beschränkte sich auf Möglichkeiten, Regenwasser zu erfassen und zu nutzen, außerdem wurde auch über die Verwendung von Grauwasser diskutiert. Der Schwerpunkt dieser Arbeit lag auf der Ausgedrung der oben genannten Techniken im Rahmen des Einfamilienwohnsitzes.

Um die in diesem Artikel vorgeschlagene bibliographische Überprüfung besser zu stützen, wurde in der Gemeinde Palhoa, $15 \mathrm{~km}$ von Florianpolis entfernt, im Bundesstaat Santa Catarina eine Fallstudie von Oliveira (2005) vorgestellt. Nach der Präsentation der Forschungsergebnisse von oliveira (2005) wurde die Diskussion mit anderen ähnlichen Studien geführt.

In den folgenden Abschnitten werden unter anderem das Untersuchungsgebiet und das Ziel sowie die Merkmale des Wohnsitzes vorgestellt, unter anderem aspekte der Fallstudie, die für diesen Artikel ausgewählt wurde.

\subsection{STUDIENBEREICH}

Die Gemeinde, die sich für die Umsetzung des Projekts entschied, war Palhoa, $15 \mathrm{~km}$ von Florianpolis zwischen der Küste und der Serra do Mar entfernt, in Meridian 48040'04" westlicher Länge und parallel 280 38' 43" südlicher Breite. Es hat eine Fläche von $323 \mathrm{~km} 2$, von denen 73,0\% des Territoriums als dauerhaftes Schutzgebiet gelten. Die Bevölkerung der Gemeinde beträgt laut IBGE (2004) 113.312 Einwohner, wie aus (PALHOA, 2004) hervorgeht. Palhoa nach Setti (1994) liegt drei Meter über dem Meeresspiegel, auf einer Meeresebene mit Mangroven, Restingas und felsigen Massiven der Serra do Mar. Das Klima, so der Autor, ist feucht mit einer durchschnittlichen Temperatur von 250C. Die durchschnittliche jährliche Niederschlagsmenge beträgt laut CLIMERH/EPAGRI 1706 mm. 


\subsection{UNTERSUCHUNGSGEGENSTAND}

Die Studie stützte sich auf zwei verschiedene Wohnhäuser und lag $2 \mathrm{~km}$ voneinander entfernt. Residence 01 hat eine Fläche von $131.36 \mathrm{~m} 2$ und Residence 02 hat eine Fläche von $143.27 \mathrm{~m} 2$. In der Residenz 01 befinden sich die Verbrauchspunkte im Bad, im Servicebereich und in der Küche. Das Badezimmer besteht aus einer Dusche, einem Waschbecken, einer Toilette mit Ventilentladung. In der Küche gibt es einen Wasserhahn auf das Waschbecken. Im Servicebereich gibt es eine Waschmaschine, die 100 Liter Wasser pro Zyklus verbraucht und einen Wasserhahn im Tank. In Der Residenz 02 befinden sich die Wasserverbrauchsstellen in drei Bädern, einer Küche und einem Essbereich. In den drei Bädern gibt es zwei Duschen, drei Wasserhähne für Waschbecken, zwei mit Mischpulten und drei sanitäre Entladungen. In der Küche gibt es einen Wasserhahn mit Mixer und im Servicebereich gibt es eine Waschmaschine mit einem Verbrauch von 80l pro Zyklus und einen Wasserhahn im Tank. In beiden Wohnhäusern wird praktisch kein Trinkwasser zum Waschen von Gehwegen, Autos oder Gartenbewässerung verwendet. Die Nutzung des von der Waschmaschine verwendeten Wassers erfolgt bereits in beiden Wohnungen und lagert es in Tanks für die spätere Wiederverwendung auf Gehwegen und Gärten.

\subsection{RAINWATER UND RE-SO WATER COLLECTION UND STORAGE SYSTEM}

\subsubsection{BESTIMMUNG DER ABDECKUNGSBEREICHE}

Um Wasser aus Regen zu speichern, ist es notwendig, die Dachflächen beider Wohnhäuser zu vermessen. Die Bestimmung dieser Gebiete wurde durch eine umfassende Analyse der Pläne der Abdeckung der vorgeschlagenen Wohnungen, die die gleiche wichtige für die Erfassung gemacht. 


\subsubsection{ROOFWATER-SAMMLUNG}

Regenwasser wird von Dachrinnen eingefangen und durch vertikale Leiter, skaliert nach NBR 10844 (ABNT, 1989), in das untere Reservoir gebracht. Dieser Standard befasst sich mit den Gebäudeinstallationen von Regenwasser.

\subsubsection{RESERVOIR FÜR REGENWASSERRÜCKNAHME}

Um das Volumen des Reservoirs zu erreichen, das Wasser aus Regen speichert, wurden die Beitragsbereiche der Abdeckung von Häusern, der tägliche Wasserverbrauch pro Einwohner, in der Niederschlagung der Region und im Koeffizienten der Nutzung von Wasser analysiert. Regen. Dieser Koeffizient zeigt den Prozentsatz an, der gespeichert wird, da der Rest für die Reinigung des Daches, der Dachrinnen und Rohre und der Verdunstung verwendet wird. Für diese Studie wird angenommen, dass $80 \%$ des auf das Dach fallenden Wassers gesammelt werden.

In jedem Wohnhaus wird es zwei Stauseen geben, eines untere, das die Lagerung von Regenwasser sammeln wird, und das obere, das verwendet wird, um die Gewässer für den Verbrauch zu verteilen. Der Boden wird Fiberglas im Gelände vergraben werden. Die Entsorgung des ersten Regenwassers erfolgt entlang des Rohres, das sich befindet, bevor das Wasser in das Reservoir gelangt. Der erste Regen wird in dieser Röhre gehalten, wodurch die meisten Verunreinigungen daran gehindert werden, das Reservoir zu erreichen. Für die Rückhaltung größerer Abfälle wird am Auslass der Dachrinnen ein flexibler PVC-Grill installiert. Am Ende des Wassereinlassrohrs im Reservoir werden eine Reduktion und ein $90^{\circ}$ Knie mit einem Durchmesser installiert, der größer ist als das Einlassrohr. Die Vergrößerung der Fläche verringert die Auswirkungen des Regenwassers, das vom Boden des Reservoirs gesammelt wird, wodurch das Rühren von abgelagerten Partikeln vermieden wird (Abbildung 03). 
Abbildung 03 - Reservoirmodell, das im Erfassungs- und Speichersystem verwendet wird.

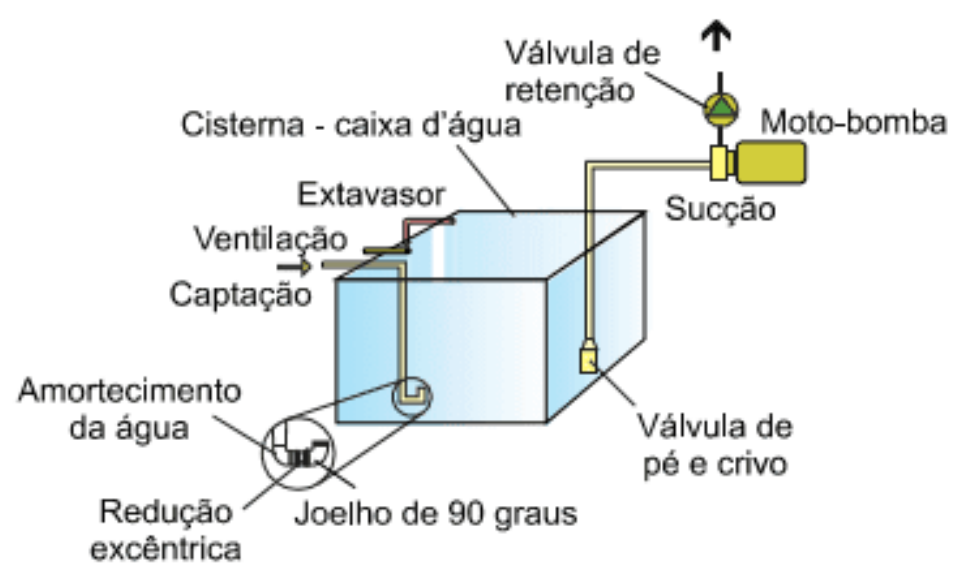

Quelle: ANA (2002) zitiert von MANUAL FIESP/CIESP (2006).

Das obere Reservoir wird über ein Motorpumpensystem mit Strom versorgt. Zwischen dem oberen Regenwasserreservoir und dem Trinkwasserreservoir sollte es ein "by pass"-System geben, das den Mangel an Regenwasser mit Trinkwasser versorgt.

\subsubsection{RESERVOIR ZUR WIEDERVERWENDUNG VON WASSER}

Die Reservoirs für die Wiederverwendung werden In beiden Wohnungen Mengen im Zusammenhang mit dem Volumen des Sekundärabwassers (Badewasser, Waschbecken, Waschmaschine und rasiertes Wasser) haben. Diese Mengen werden durch eine Am Ort des Wasserverbrauchs durchgeführte Erhebung überprüft. In jedem Wohnhaus wird es zwei Reservoirs geben: eine niedrigere implantierte für die Wasserspeicherung und eine überlegene nur für die Verteilung des Verbrauchs. Das untere Reservoir wird Glasfaser und im Boden vergraben werden. Vor dem Erreichen des Reservoirs werden Sekundärabwässer einer Sandentfernungsbox für die Rückhaltung des Abfalls und einem Pflanzenbehandlungssystem namens Wurzelzone unterzogen. Nach der Lagerung des Wassers bereits mit ordnungsgemäßer Behandlung, wird es zum oberen Reservoir durch ein Motorradpumpensystem gefahren werden und dann die Einsatzstellen zuführen. 


\section{ERGEBNISSE}

Für das von Oliveira (2005) vorgestellte Projekt, bei dem regenwasser eingefangen, gelagert und verteilt und wiederverwendet wird, wurde in der Gemeinde Palhoa eine Untersuchung der Endverwendung dieses Wassers durchgeführt. Aus der Bestimmung dieser Endverwendungen kann die für die Verwendung von Regenwasser notwendige Wassermenge und das Volumen des Sekundärabwassers bestimmt werden, das für seine Wiederverwendung zur Verfügung steht. Nach der Bestimmung dieser Volumina werden die Systeme für Regenwasser und für die Wiederverwendung von Grauwasser skaliert, und dann wird die wirtschaftliche Analyse durchgeführt, um die Lebensfähigkeit der implantierten Systeme zu bestimmen.

\subsection{PUNKTE HÖHERER VERBRAUCH IM WOHNORT 01}

Nach der Korrektur durch die Sensitivitätsmethoden, der Ort, wo der höchste Wasserverbrauch war die Dusche mit 32,8\% des Gesamtverbrauchs der Residenz, gefolgt von der Toilette mit 30,4\% und der Küchenhahn mit 28,0\%, nach Tabelle 01.

Tabelle 01 - Beschreibung des Wasserverbrauchs an den Wohnorten 01

\begin{tabular}{|c|c|c|c|c|c|}
\hline \multirow{2}{*}{ Aparelho } & \multicolumn{4}{|c|}{ Consumo (litros) } & \multirow[b]{2}{*}{$\%$} \\
\hline & $\mathrm{H}_{1}$ & $\mathbf{M}$ & $\mathbf{H}_{2}$ & Total & \\
\hline Chuveiro & 1528,9 & 1662,6 & 2092,8 & 5284,3 & 32,8 \\
\hline Lavatório & 92,8 & 132,1 & 73,8 & 298,7 & 1,9 \\
\hline Vaso Sanitário & 1465,1 & 2289,6 & 1138,6 & 4893,2 & 30,4 \\
\hline Barba & 75,6 & - & 55,2 & 130,8 & 0,8 \\
\hline Máquina Lavar Roupa & 333,3 & 333,3 & 333,3 & 1000,0 & 6,2 \\
\hline Torneira Cozinha & 1505,0 & 1505,0 & 1505,0 & 4515,0 & 28,0 \\
\hline TOTAL & & & & 16122,0 & 100,0 \\
\hline
\end{tabular}

Quelle: Oliveira, 2005 


\subsection{PUNKTE HÖHERER VERBRAUCH IM WOHNORT 02}

Im Wohnort 02 wurde nach der Korrektur auch der höchste Verbrauch in der Dusche beobachtet, mit 45,6\% des Gesamtverbrauchs der Residenz, gefolgt von der Toilette mit 25,6 \% und der Spüle in der Küche mit 13,5\%, wie in Tabelle 02 dargestellt.

Tabelle 02 - Beschreibung des Wasserverbrauchs an den Wohnorten 02

\begin{tabular}{|c|c|c|c|c|}
\hline \multirow{2}{*}{ Aparelho } & \multicolumn{3}{|c|}{ Consumo (litros) } & \multirow[b]{2}{*}{$\%$} \\
\hline & $\mathbf{M}$ & $\mathbf{H}$ & Total & \\
\hline Chuveiro & 1339,2 & 1762,8 & 3102,0 & 45,6 \\
\hline Lavatório & 112,0 & 66,2 & 178,3 & 2,6 \\
\hline Vaso Sanitário & 986,7 & 755,5 & 1742,2 & 25,6 \\
\hline Barba & - & 300,0 & 300,0 & 4,4 \\
\hline Máquina Lavar Roupa & 280,0 & 280,0 & 560,0 & 8,2 \\
\hline Torneira Cozinha & 460,8 & 460,8 & 921,6 & 13,5 \\
\hline TOTAL & & & 6804,0 & 100,0 \\
\hline
\end{tabular}

Quelle: Oliveira, 2005

\subsection{FINAL USES FÜR RAINWATER UND WASSER RESO}

Zur Bestimmung des Tagesvolumens für die Wiederverwendung von Wasser und des für die Verwendung von Regenwasser erforderlichen Tagesvolumens wird durch die endgemäße Verwendung von Wasser nach Korrektur im geschätzten Verbrauch ermittelt. Die Tabellen 03 und 04 berücksichtigen die für die Wiederverwendung verbrauchte Wassermenge sowie die für die Versorgung durch Wiederverwendung erforderliche Menge und das für die Verwendung von Regenwasser erforderliche Volumen. Die benötigte Wassermenge, die als Wiederverwendung zur Verfügung steht, wurde aus dem Verbrauch in der Dusche, Waschbecken, Wasser verwendet, um rasieren und in der Waschmaschine, insgesamt, für Wohnort 01, etwa 240 Liter, was $41,9 \%$ des Verbrauchs entspricht Gesamtwohnsitz. Mit dem Wiederverwendungswasser können Sie nur die Toilette versorgen, mit einem täglichen Bedarf von 175,0 Litern, etwa $30 \%$ des Verbrauchs der Residenz. Daher steht genügend Wasser zur Verfügung, um die Toilette zu versorgen. Mit Regenwasser 
können Sie die Toilette und Waschmaschine versorgen. In Residenz 01, gibt es einen täglichen Bedarf von 210.5 Liter, etwa 36.6\% des Gesamtverbrauchs der Residenz.

Tabelle 03 - Verbrauch zur Wiederverwendung und Verwendung von Regenwasser in Residenz 01.

\begin{tabular}{lccc|ccc|ccc}
\hline \multirow{4}{*}{ Aparelhos } & \multicolumn{10}{c}{ Consumo } \\
\cline { 2 - 10 } & \multicolumn{2}{c}{ Reúso - Disponível } & \multicolumn{2}{c}{ Reúso - Necessário } & \multicolumn{3}{c}{ Água Chuva } \\
\cline { 2 - 11 } & $\begin{array}{l}\text { Diário } \\
\text { (litros) }\end{array}$ & $\begin{array}{l}\text { Mensal } \\
\text { (litros) }\end{array}$ & $\%$ & $\begin{array}{l}\text { Diário } \\
\text { (litros) }\end{array}$ & $\begin{array}{c}\text { Mensal } \\
\text { (litros) }\end{array}$ & $\%$ & $\begin{array}{l}\text { Diário Mensal } \\
\text { (litros) }\end{array}$ & $\begin{array}{c}\text { (litros) } \\
\%\end{array}$ \\
\hline Chuveiro & 188,7 & 5284,3 & 32,8 & - & - & - & - & - & - \\
Lavatório & 10,7 & 298,7 & 1,9 & - & - & - & - & - & - \\
Vaso Sanitário & - & - & - & 174,8 & 4893,3 & 30,4 & 174,8 & 4893,3 & 30,4 \\
Barba & 4,7 & 130,8 & 0,8 & - & - & - & - & - & - \\
Máquina Lavar Roupa & 35,7 & 1000,0 & 6,2 & - & - & - & 35,7 & 1000,0 & 6,2 \\
\hline TOTAL & $\mathbf{2 3 9 , 8}$ & $\mathbf{6 7 1 3 , 8}$ & $\mathbf{4 1 , 9}$ & $\mathbf{1 7 4 , 8}$ & $\mathbf{4 8 9 3 , 3}$ & $\mathbf{3 0 , 4}$ & $\mathbf{2 1 0 , 5}$ & $\mathbf{5 8 9 3 , 3}$ & $\mathbf{3 6 , 6}$ \\
\hline
\end{tabular}

Quelle: Oliveira, 2005

Tabelle 04 - Verbrauch zur Wiederverwendung und Verwendung von Regenwasser in Residenz 02.

Quelle: Oliveira, 2005

\subsection{FINAL SAVINGS MIT DER CAPTURE, STORAGE UND TREATMENT OF RAINWATER UND RE'SO}

Tabelle 05 zeigt die Einsparungen beim Trinkwasser, die sich auf die Nutzung des Wassernutzungssystems durch Regen, die Einführung des Wiederverwendungswassersystems und die Durchführung von Regenwasser und wiederverwenden zusammen, in den Häusern 01 und 02, in Währung beziehen ( und in Prozent.

In Residenz 01 gibt es eine jährliche Einsparung mit Trinkwasser von 35,5\% durch die Einführung des Regenwassernutzungssystems und etwa $30,4 \%$ bei der Nutzung des Wiederverwendungssystems. Durch die Umsetzung der Regenwasser- und Wiederverwendungsanordnung ergaben sich Einsparungen von $36,4 \%$. 
Für die Residenz 02, die Einführung eines Systems der Nutzung von Regenwasser, wurde eine Wirtschaft mit Trinkwasser von 33,6\% und mit der Umsetzung der Wiederverwendung, 25,6\%. Die Gesamtwirtschaft mit beiden Fangformen betrug 33,8 $\%$.

Tabelle 05 - Jährliche Einsparungen in den Residenzen 1 und 2.

\begin{tabular}{lcccc}
\hline \multirow{2}{*}{\multicolumn{1}{c}{ Economia }} & \multicolumn{4}{c}{ Economia } \\
\cline { 2 - 5 } & \multicolumn{2}{c}{ Residência 1 } & \multicolumn{2}{c}{ Residência 2 } \\
\cline { 2 - 5 } & $\mathbf{R}$ R & $\mathbf{\%}$ & \% \\
\hline Economia anual - água de chuva & 117,10 & 35,5 & 46,75 & 33,6 \\
Economia anual - reúso de água & 100,05 & 30,4 & 35,60 & 25,6 \\
Economia anual - água de chuva + reúso & 119,84 & 36,4 & 47,01 & 33,8 \\
\hline
\end{tabular}

Quelle: Oliveira,2005

\section{DISKUSSION}

Für das in diesem Artikel angesprochene Projekt in der Stadt Palhoa verwendete das System einfache Komponenten, die alle Grundlegendenanforderungen erfüllten und die nicht nur eine einfache Methode sind, sondern auch Einsparungen für die Bewohner generieren können, bei denen die Anfangsinvestition gering war und die Rückkehr in kurzer Zeit. Die Forschung vergleicht auch die gleiche Methode, die in zwei verschiedenen Einheiten angewendet wird, die darauf abzielt, die Baumethoden und ihren Beitrag zur Sammlung und Speicherung von Wasser zur Wiederverwendung zu ermitteln.

Andere Autoren etablieren unterschiedliche Systeme für die Erfassung und Behandlung von Grau- und Regenwasser, wobei die Wirtschaftlichkeit je nach den von den einzelnen Modellsystemen festgelegten Investitionen und auch nach der Effizienz des Systems variiert.

Nach Silveiras Arbeit (2008) umfasst die "Europalinie", die auf einer deutschen Regenwasseraufbereitungserhebung basiert, Systeme, die den häuslichen und externen Gebrauch liefern und auch Bereiche mit größerer Erfassung bedienen. Es ist ideal für Wohnhäuser, die sich noch in der Bauphase befinden, da es eine bessere 
Integration zwischen Trink- und Regenwassersystemen ermöglicht. Diese Methode nach Angaben des Forschers wird eine Pumpe verwenden, zusätzlich zu anderen Zubehör wie Wasserbremse (um den Wirbelwind in der Zisterne zu reduzieren), schwimmenden Filter, um eine höhere Qualität des gefangenen Wassers und MultiSiphon zu ermöglichen, um insektenfrei zu lassen in das System eingeben. Die Reservoirdimensionierung für die Lagerung wird die gleiche sein wie die, die bei den Arbeiten in der Stadt Palhoa durchgeführt wird, wo sie durch die Verbrauchsprognose, die Erfassungsbasis und die Trockenzeit für den Standort definiert wird. Es kann auch das Regenwassersystem mit dem Wasser des öffentlichen Versorgungsnetzes verbinden, so dass in den Momenten des Wassermangels die Integration zwischen den beiden Systemen Qualität und Versorgung garantiert, so Abbildung 04.

Abbildung 04: Modell der Europalinie adressiert von der mestranda Bruna Quick da Silveira

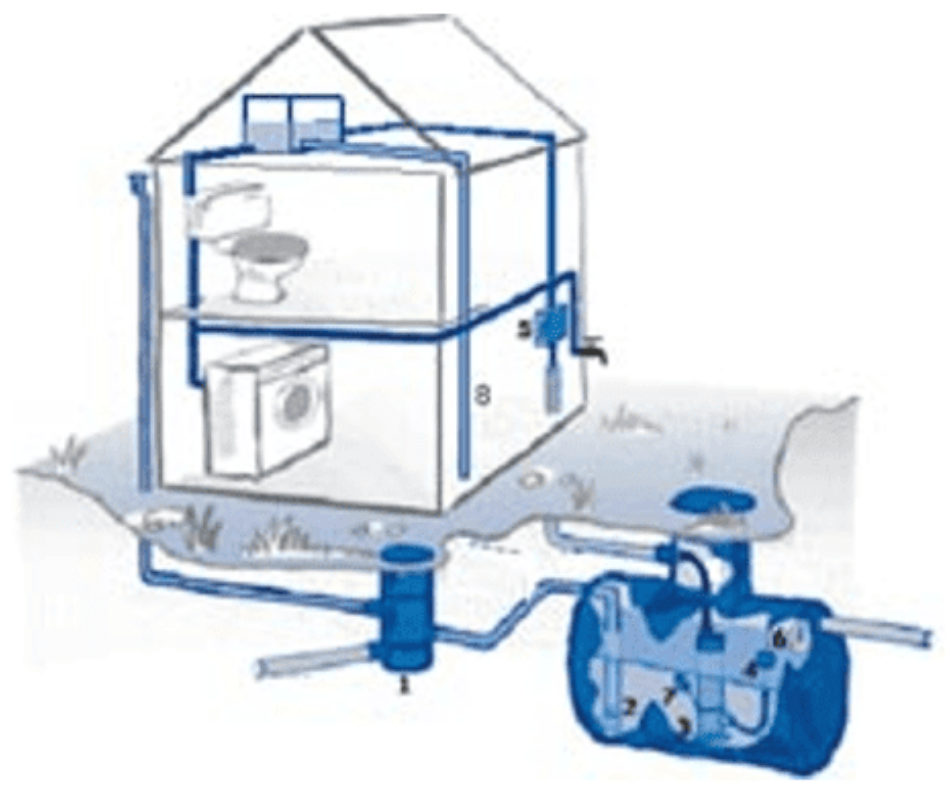

1.Filtro tipo vortex

2. Freio d'água

3. Bomba submersivel

4. Filtro flutuante

5. Central de controle/ interligação com rede pública

6. Multisifäo

7. Bóia de nível

8. Alimentação dos Pontos de Consumo a partir da Caixa d'água superior

Quelle: AQUASTOCK, 2008. 
Die beiden konfrontierten Systeme sind effizient, nutzen aber unterschiedliche Möglichkeiten, um das gesammelte Wasser zu erfassen, zu behandeln und zu verteilen, wo jede Methode ihre Besonderheit hat. Die Effizienz beider ist zufriedenstellend, was die beiden Systeme üblich und wirtschaftlich macht, da sie als bestimmender Faktor für die Wahl der am besten geeigneten anfänglichen Investition, die jeder verlangen wird, da die in der Stadt Palhoa die einfachste Methode verwendet wird, die endet eine Anfangsinvestition, die kleiner ist als die von der Mestranda, die als "Europe Line"-System bezeichnet wird.

\section{FAZIT}

Aus dem Ziel dieses Artikels kann man erkennen, dass das Interesse der Gesellschaft gering ist, wenn das Thema auf der Tagesordnung die Installation eines Systems ist, das in der Lage ist, das zuvor verworfene Wasser wiederzuverwenden. Dies liegt daran, dass es ein gewisses Vorurteil bei der Wiederverwendung von Wasser aus Haushaltsabwässern gibt und auch auf das höhere Kontaminationsrisiko im Zusammenhang mit dem Instandhaltungssystem zurückzuführen ist, das Schäden an der Gesundheit direkter oder indirekter Nutzer des Systems fördern kann. Eine geeignete und bewusst betriebene Wiederverwendungsmethode erhöht die Effizienz und reduziert jede Art von Kontamination, die vom Bediener oder System als Ganzes auftreten kann.

Das in diesem oder anderen auf der Welt verfügbare Erfassungsmodell erfordert sehr hohe Erstinstallationskosten, aber auf lange Sicht muss es Einsparungen in der Wassermenge in der Residenz, sowie in der Qualität des Wassers, das empfangen wird, denn bei der Behandlung und Wiederverwendung des Wassers, das einmal in die Natur zurückkehrte, wird es die Erhaltung der Qualität dieses Gutes geben, das neben endlicher Bedeutung für das Wohlbefinden und die Lebensqualität des Menschen ist. 


\section{REFERENZEN}

ABNT - Associação Brasileira de Normas Técnicas. NBR 10844 - Instalações Prediais de Águas Pluviais. Rio de Janeiro (1989).

ABNT Associação Brasileira de Normas Técnicas. NBR 5626 - Instalações Prediais de Água Fria. Rio de Janeiro (1998).

ANA - Agência Nacional de Águas. A Evolução da Gestão dos Recursos Hídricos no Brasil / The Evolution of Water Resources Management in Brazil. Brasília; ANA, 2002.

AQUASTOCK - Água da Chuva. Sistema de Reaproveitamento da Água da Chuva. Disponível em: <http://www.engeplasonline.com.br> Acesso em: 21/08/2008

BIO: Revista Brasileira de Saneamento e Meio Ambiente. Água: o ouro azul do século XXI. Rio de Janeiro, RJ, v.11, n. 21, jan./mar. 2002

BIO: Revista Brasileira de Saneamento e Meio ambiente. Centronetwork. Rio de Janeiro, $\mathrm{n}^{\circ}$ 21. Ano XI. 2002. p.50

BRAGA, BENEDITO et al. Introdução à Engenharia Ambiental O desafio do desenvolvimento sustentável. 2ª edição. São Paulo: Pearson Prentice Hall, 2005.

CAUBET, Christian Guy. A água doce nas relações internacionais,ano 2006.

FIORI, Simone. Avaliação Qualitativa E Quantitativa Do Potencial De Reuso De Água Cinza Em Edifícios Residenciais Multifamiliares. Dissertação de Mestrado da Universidade de Passo Fundo (RS), 2005. Disponível: <http//www.usp.br>. Acesso em 12/11/2016.

GONÇALVES, R. F. (Coord.). Conservação de água e energia em sistemas prediais e públicos de abastecimento de água. Rio de Janeiro: ABES, 2009 
HESPANHOL, I. Potencial de Reúso de Água no Brasil: Agricultura, Indústria, Municípios, Recarga de Aquíferos. Recursos Hídricos: APRH, Portugal, v. 23, n.2, p.43-65, nov. 2002.

LIBÂNIO, M. Fundamentos de qualidade e tratamento de água, $3^{\circ}$ edição Campinas: Átomo, 2010.

MARIANE, Aline -Construtoras adotam sistemas de reuso e aproveitamento de água de chuva, além de métodos de construção seca, para contornar falta de água nas obras. Disponível em: http://construcaomercado.pini.com.br/negocios-incorporacaoconstrucao/161/artigo333851-1.aspx >Dez,2014. Acesso em: 12 Dez.2016.

MAY, Simone. Conservação e Reúso de Água em Edifícios: Reúso De Águas Cinzas e Aproveitamento De Águas Pluviais Para Consumo Não Potável. Escola Politécnica da Universidade de São Paulo (USP),2009.

MAYOK, SIMONE. Caracterização, tratamento e reuso de águas cinzas e aproveitamento de águas pluviais em edificações. São Paulo, junho, 2009.

MIERZWA, J.C; VERAS,L.R; SILVA,M.C. Avaliação do Desempenho de Membrana de Ultrafiltração para Tratamento de Água Potável. VIII Seminário IberoAmericano. São Paulo: 2005.

OLIVEIRA, N. M.; SILVA, M. P. da.; CARNEIRO, V. A. Reúso da água: um novo paradigma de sustentabilidade. Revista Gestão e Desenvolvimento em ContextoGEDECON Edição Especial - Porangatu, v.2, n.1, p.146-157, jan. /jul. 2013.

OLIVEIRA, Sulayre Mengotti de. Aproveitamento da água da chuva e reúso de água em residências unifamiliares: estudo de caso em Palhoça - SC, junho de 2005.

OPAS (2001) Água e Saúde.Relatório.www.opas.org.br/sistema/fotos/agua.pdf. $<$ Acessado em abril de 2017> 
PALHOÇA, Prefeitura Municipal de Palhoça. Disponível em: http://palhoça.sc.gov.br, acessado em novembro de 2004.

PROSAB, Uso Racional da Água em Edificações-Ricardo Franci Gonçalves (Coord.). Rio de Janeiro : ABES, 2006

SETTI, A. A. A Necessidade do Uso Sustentável dos Recursos Hídricos. IBAMA, Brasília, 1994.

SICKERMANN, Jack M. Gerenciamento Sustentável das Águas de Chuva: imprescindível para o futuro das grandes cidades do Brasil. Piauí, 2005. 9p.

SILVEIRA, Bruna Quick da. REUSO DA ÁGUA PLUVIAL EM EDIFICAÇÕES RESIDENCIAIS, janeiro de 2008.

TOMAZ, P. Economia de água para empresas e residências: um estudo atualizado sobre o uso racional da água. $2^{\circ}$ Edição. São Paulo: Navegar Editora, 2005.

VESENTINI, J. W. Brasil, sociedade e espaço. 7. ed. São Paulo: Ática, 1999.

ZAMPIERON, Sônia Lúcia Modesto; VIEIRA, João Luiz de Abreu. "Poluição da Água". Disponível em: <http://educar.sc.usp.br/biologia/textos/m_a_txt5.html>

Eingereicht: November 2019.

Genehmigt: Januar 2020. 